\title{
Sensorimotor deficits in a unilateral intrastriatal 6-OHDA partial lesion model of
}

\section{Parkinson's Disease in marmoset monkeys.}

Andisheh Eslamboli, Harry F Baker, Rosalind M Ridley, Lucy E Annett ${ }^{1}$

Department of Experimental Psychology

Downing Street

Cambridge CB2 3EB

\author{
${ }^{1}$ Psychology Department \\ University of Hertfordshire \\ College Lane, Hatfield
}

Hertfordshire AL10 9AB

Running title: Partial lesion model of Parkinson's disease in monkeys

Address for correspondence: Dr HF Baker

Innes Building

School of Veterinary Medicine

Madingley Road

Cambridge CB3 OES

Tel: 01223339015

Fax: 01223339014

E-mail: hfb22@cam.ac.uk 


\begin{abstract}
Animal studies investigating the efficacy of neurotrophic factors as treatments for Parkinson's disease (PD) ideally require partial dopamine (DA) lesion models. The intra-striatal 6hydroxydopamine (6-OHDA) lesion model may be suitable for this purpose. Although this model has been well characterized in rodents, it has not previously been used in monkeys. The goal of the present study was to characterize the behavioural effects of unilateral injections of 6-OHDA in the basal ganglia of common marmoset monkeys (Callithrix jacchus). Cell counts from tyrosine hydroxylase immunochemistry five months postlesion revealed DA cell loss in the substantia nigra on the lesioned side of $\sim 46 \%$ relative to the unlesioned side. 6-OHDA lesioned monkeys showed a variety of behavioural deficits. Apomorphine induced rotation and simple sensorimotor measures (head position bias and PD disability rating score) were most affected by the lesion. The largest deficits were seen at one or two weeks postsurgery but had recovered by week ten. 6-OHDA lesioned monkeys took longer to complete a more complex sensorimotor staircase task. At 3.5 months postlesion, 6-OHDA monkeys also showed deficits on an object retrieval task designed to measure sensorimotor planning and skilled hand use. $\alpha$ Methyl-p-tyrosine, a tyrosine hydroxylase inhibitor, reinstated those deficits which had undergone recovery in the lesioned animals and also exacerbated the deficits on the staircase task. This model has potential in assessing treatments for PD aimed at curtailing disease progression such as continuous delivery of neurotrophic factors.
\end{abstract}

Key words: Marmoset; Monkey; Parkinson’s disease; 6-Hydroxydopamine; Dopamine; Basal ganglia; Substantia nigra; Caudate; Accumbens; Putamen 


\section{Introduction}

Parkinson's disease (PD) is one of the most common debilitating neurodegenerative diseases, with 1-2 individuals affected for every $1000(9,16,35)$. The disease manifests itself as bradykinesia, rigidity and resting tremor (20) with patients frequently presenting cognitive deficits $(6,42)$. The neuropathology underlying PD centres primarily on a severe, but subtotal, loss of dopamine (DA) cells in the substantia nigra pars compacta (SNpc) $(12,25)$.

A variety of strategies has been developed in the search for an effective treatment for PD. These strategies can be broadly classified as those attempting to control the symptoms of the disorder and those attempting to halt or reverse disease progression (17). The mainstay of the former approach includes the use of dopamine replacement drugs such as L-DOPA (54). More invasive techniques have also been attempted, including brain stimulation (15) and intracerebral transplantation of foetal dopaminergic cells $(7,23)$. Unfortunately, such treatments fail to halt the progress of the disease and are not without risk of serious side effects (e.g. L-DOPA induced dyskinesias) (44).

Within the last decade, attention has been directed at methods designed to halt or reverse disease progression. Such strategies include attempts to rescue damaged neurones with the aid of neurotrophic factors, such as glial cell line-derived neurotrophic factor (GDNF) (4). Strategies involving GDNF, in particular, seem promising since GDNF has been shown to be expressed in the developing and adult striatum $(26,40)$. Furthermore, it has been shown to increase survival of DA neurones in vitro and in vivo when given before or after mechanical axotomy $(3,57)$ or treatment with neurotoxins targeting DA neurones $(19,31,32,59)$. 
Studies investigating neurotrophic factors such as GDNF pose a particular challenge regarding the appropriate model needed to test its efficacy. In rodents, the in vivo model most commonly used in PD research involves the unilateral injection of 6-hydroxydopamine (6-OHDA) directly into the substantia nigra or the medial forebrain bundle (52). These procedures produce DA depletions in the terminal striatal areas which are almost complete $(37,41)$. In order to assess the neuroprotective efficacy of neurotrophic factors, however, a partial lesion animal model is required (5). Ideally, such a model should parallel the gradual degeneration of DA neurones characteristic of PD where a small number of DA neurones remain at the end-stage of the disease $(12,25)$. These remaining groups of neurones are the targets for neuroprotective strategies (33). Unfortunately, it is difficult to produce standardized partial lesions by making sub-total lesions within the substantia nigra or medial forbrain bundle (5). An alternative approach has been developed in rats in which 6-OHDA is injected directly into the terminal areas in the striatum. In the past few years, this intrastriatal 6-OHDA model in rats has been well characterized and been shown to produce suitable partial DA lesions $(2,29,34,43)$. In this model there is a gradual degeneration, in which the first stage of rapid cell loss (one to four weeks post-injection) is followed by protracted cell loss continuing for at least four months (51). The majority of rat studies showing the neuroprotective and neuroregenerative capacity of GDNF have used this particular partial lesion model (10, 28, 30, 31, 36, 48-50, 59). For example, Kirik and colleagues (31) have shown that administration of a modified adeno-associated viral vector (AAV) expressing GDNF to rats prior to intrastriatal injection of 6-OHDA was able to protect dopamine cells against the toxic insult and also to result in preservation of function.

Before treatments using viral vectors expressing GDNF progress to clinical use their safety and efficacy in monkeys should be assessed as an intermediate step between rodent studies and 
patient trials for a number of reasons. For example, the putamen and caudate nucleus form a continuous structure in rodents whereas they are discrete structures in humans and monkeys. Also, there are immunological and pharmacokinetic differences between primates and rodents.

Although there have been monkey studies in which GDNF has been evaluated, they have made use of the MPTP model of PD $(11,19,21,32,60)$. Since the majority of rodent studies rely on the intrastriatal 6-OHDA model, we thought it was important to investigate the efficacy of GDNF in monkeys based on the same model. This would allow for direct comparison with the majority of rodent studies testing GDNF. However, the 6-OHDA partial model has not yet been characterised in monkeys. The goal of the present study was to characterize the behavioural effects of 6-OHDA lesions within the dopaminergic terminal striatal area in the common marmoset monkey.

Methods

Animals and Surgery

All procedures were carried out under a Project Licence in accordance with the U.K. Animals (Scientific Procedures) Act 1986. Twelve adult common marmosets (Callithrix jacchus), 7 males and 5 females, were used. All were laboratory-bred, weighed 320-470g, and were aged 22-35 months at the start of the experiment. All the monkeys were housed with a cage-mate of similar age. Six monkeys (4 males, 2 females) were given 6-OHDA injections unilaterally, with 9 injections into the striatum (caudate, accumbens, putamen). Six monkeys ( 3 males, 3 females) were given saline control injections at the same sites. Attempts were made to ensure that each pair of cage-mates were given unilateral injections in opposite hemispheres. Thus 3 animals in each group had injections in the left hemisphere and 3 had injections in the right 
hemisphere. The coordinates, derived from the stereotaxic atlas of Stephan et al (53), are shown in Table 1.

For surgery, monkeys were anaesthetized with alphaxalone-alphadolone (Saffan; ScheringPlough Ltd, Welwyn Garden City, UK; $1.5 \mathrm{ml} / \mathrm{kg}$ given intramuscularly). Fifteen minutes later the monkeys were injected with pargyline (Sigma, St Louis, MO, USA; 50mg/kg, intraperitoneally), a monoamine oxidase inhibitor which prevents the metabolism of 6-OHDA, thus prolonging its neurotoxic effects. A supplementary dose of $0.3 \mathrm{ml}$ Saffan was given during surgery if necessary. 6-OHDA Hbr (Sigma, St Louis, MO, USA; 4mg/ml free base weight dissolved in $0.01 \%$ ascorbate/saline) was freshly prepared and stored on ice just before use. Each injection of $2 \mu 16$-OHDA was made at a rate of $0.5 \mu 1 / \mathrm{min}$ using a $29 \mathrm{~g}$ injection needle which was left in place for a further four minutes after each injection had been made. Control monkeys underwent the same procedures with saline injections. Following surgery the monkeys were given an analgesic (Finadyne; Schering-Plough Animal Health, Mildenhall, UK; $0.1 \mathrm{mg} / \mathrm{kg}$, subcutaneously) and kept in a warm incubator until well enough to be returned to their home cage, usually the day following surgery.

Histology

When all behavioural testing was finished, the monkeys were perfused for histological analysis of the brains. The monkeys were premedicated with $0.05 \mathrm{ml}$ ketamine (Vetalar; SheringPlough, Welwyn garden City, UK; 100mg/ml, intramuscularly) and deeply anaesthetised with $0.6 \mathrm{ml}$ sodium pentobarbitone $(200 \mathrm{mg} / \mathrm{ml}$, intraperitoneally) prior to being perfused. Monkeys were perfused transcardially with $500 \mathrm{ml} 0.1 \mathrm{M}$ phosphate-buffered saline pH7.4 (PBS), followed by $1000 \mathrm{ml}$ of $4 \%$ paraformaldehyde in PBS. Following perfusion, the brains were 
removed and placed in $4 \%$ paraformaldehyde solution for 24 hours, after which they were transferred to $30 \%$ sucrose solution in PBS for 4 days at $4^{\circ} \mathrm{C}$.

Sections were cut at $60 \mu \mathrm{m}$ thickness and 1 in 6 sections was stained with cresyl violet. One in 6 sections was stained for tyrosine hydroxylase (TH). The staining for TH was carried out on free-floating sections and was done separately for the cell bodies in the $\mathrm{SN}$ and the terminals in the striatum. For the cell bodies, sections were treated for 5 min with $10 \%$ methanol/10\% hydrogen peroxide in distilled water, to remove endogenous peroxidase. Sections were then washed in Tris-buffered saline, pH7.4 (TBS) and then pre-incubated in 3\% normal goat serum (NGS; Dako, UK) for one hour in TBS containing 0.2\% Triton-X100 (TXTBS), to reduce non-specific staining. The sections were then incubated for 3 days at $4{ }^{\circ} \mathrm{C}$ in polyclonal tyrosine hydroxylase antibody (1:3000, Institute Jacques Boy, Rheims, France) in 1\% NGS in TXTBS. Sections were then washed in TBS and incubated in biotinylated anti-rabbit immunoglobulin (Dako, UK; 1:200 in 1\% normal goat serum in TBS). Sections were then washed in TBS and incubated for 2 hours in a streptavidin-biotin-peroxidase kit (Dako, UK; in 1\% NGS in TBS). After washes in TBS and Tris buffer (0.05M Tris/HCL, pH 7.4), the sections were treated with 3,3'-diamino benzidine (1\% in Tris buffer). The sections were washed in Tris buffer, mounted on $1 \%$ gelatin-coated microscope slides and left to dry over night. After rehydration and dehydration through graded alcohols, the slides were cleared in xylene and coverslipped using DPX mountant.

TH staining of the striatal terminals was carried out as above with a few modifications. To remove endogenous peroxidases, the sections were incubated for 10 minutes in $10 \%$ methanol/3\% hydrogen peroxide. Potassium phosphate buffered saline (KPBS) was used instead of TBS for all rinses and solutions. The sections were not preincubated in NGS, but 
for all other steps NGS was used at $2 \%$ rather than $1 \%$. The sections were incubated overnight at room temperature in a different polyclonal tyrosine hydroxylase antibody $(1: 2000$, Pel-Freeze, Rogers, AR, USA; in $2 \%$ NGS in $0.25 \%$ triton in KPBS). The sections were incubated in the streptavidin-biotin-peroxidase kit for one hour rather than two.

Quantification of TH-immunoreactive cells (TH-IR) in the SNpc

Prior to analysis, the borders of the SNpc were defined. In the anterior sections, the pars compacta cells were the only TH cells visible. In the central and caudal sections the border between the ventral tegmental cell group and the pars compacta region was just lateral to the roots of the third nerve. At the most caudal level, the pars compacta was defined as the dense TH cell group ventral to the medial lemniscus and the retrorubral area. The TH positive cells in the pars retriculata (and therefore dorsal to the cerebral peduncle) were included in the cell counts. This definition led typically to 7-8 sections per monkey being measured.

The number of TH-IR cells was quantified using the unbiased stereological method of the optical fractionator (58), as outlined in Kirik et al (29). Sampling was done using an Olympus CAST-Grid system (Olympus Danmark A/S, Denmark). After the region of interest was delineated for each slide, the first sampling counting frame was randomly placed over the region of interest. The counting frame was then systematically moved through the counting area until the whole area was sampled. The size of the counting frame was predetermined and a frequency was chosen so that between 100 and 200 cells would be counted per SNpc. The total number of cells was estimated based on the optical fractionator formula (58).

Quantification of striatal TH-IR fibre density 
Optical densitometry readings were made of the TH-IR fibres in the striatum using a NIH 1.60 Image program on a Macintosh 7100/66 computer. The computer was connected to an MT172 CCD video camera and a constant illumination table. Readings were made from nine rostro-caudal levels of the striatum one millimetre apart starting from AP13.5 and moving caudally, according to the atlas of Stephan et al (53). Readings from the corpus callosum were used to correct for non-specific background density levels.

\section{Behavioural Tests}

The person undertaking all the behavioural assessment (AE) was unaware of whether the monkeys had had 6-OHDA or saline injections.

Disability rating scale: This rating scale was used to quantify a variety of behavioural signs which could be regarded as 'parkinsonian'. Although the current experiment involved monkeys with unilateral lesions, we have previously found that non-lateralised measures can be sensitive to unilateral lesions (LEA, personal observations). The degree to which monkeys presented parkinsonian features in both spontaneous and induced behaviour was recorded. The readings were made in the home room when other non-experimental monkeys in the room were active (e.g. mid-morning or mid-afternoon, but not immediately after feeding). For spontaneous behaviours, the observer stood approximately $2 \mathrm{~m}$ in front of the cage, and scored the behaviour in 3 separate one minute intervals. The following were assessed: 1) akinesia, 2) climbing, 3) abnormal posture and 4) tremor. The monkeys were given a score from 0 to 3 for each of these behaviours for each minute, with 3 representing the most impairment and 0 being normal behaviour. For induced behaviour, the observer tempted each monkey 3 times with a 
small piece of marshmallow $\left(\sim 3 \mathrm{~mm}^{3}\right)$ and on each occasion the monkey was given a score of 0 to 3 on: 1) clumsiness, 2) bradykinesia, and 3) poor balance. For each monkey the overall score consisted of the total scores for spontaneous and induced behaviours to give a score of 63 for maximum impairment. Monkeys were assessed before lesion and at one, 2, 6, and 10 weeks after the lesion.

Head position bias: Monkeys with unilateral dopaminergic lesions tend not to initiate movement into their contralesional side and as a result show a postural bias towards the ipsilesional side. The head bias score was a quantification of the time the monkeys spent with the head turned toward the lesioned side. The observer rated the head position with respect to the rest of the body (i.e. left, right, or straight ahead) every second for 3 minutes. This was repeated on 4, usually consecutive, days. For each monkey the final score consisted of the average number of seconds per day spent with the head turned ipsilesionally minus the number of seconds spent with the head turned contralesionally. Monkeys were assessed before lesion and at one, 2, 6, and 10 weeks after the lesion.

Rotation: The monkeys were assessed for both spontaneous and drug-induced rotation. For spontaneous rotation each monkey was placed in a Plexiglass box $(\sim 20 \mathrm{~cm}$ wide $\mathrm{x} 15 \mathrm{~cm}$ deep $\mathrm{x} 15 \mathrm{~cm}$ high) in a test room and videotaped for 30min, during which time the experimenter left the room. For amphetamine-induced rotation each monkey was injected intramuscularly with amphetamine (Sigma, St Louis, MO, USA; $0.5 \mathrm{mg} / \mathrm{kg}$ dissolved in saline) 30min before being videotaped for 30min. For apomorphine-induced rotation each monkey was injected intramuscularly with apomorphine (Sigma, St Louis, MO, USA; 0.05mg/kg dissolved in $0.1 \%$ ascorbate in saline) immediately before being videotaped for 60min. Monkeys were assessed 
before lesion, at one week after the lesion for spontaneous rotation, and at 2, 6, and 10 weeks after the lesion for spontaneous and drug-induced rotation.

Staircase task: The staircase task is analogous to the 'staircase test' used for rats $(38,39)$ to assess skilled forelimb reaching. For this task, monkeys were tested individually in their home cages after their cage mate had been removed for the duration of the test session. The test apparatus was fixed to the front of the cage and the monkey was required to reach through a vertical slot on the left or right side of a Plexiglass apparatus in order to retrieve syrup-soaked bread pieces $\left(\sim 5 \mathrm{~mm}^{3}\right)$ arranged on a series of five steps ascending towards the midline. An angled inner wall ensured that the monkey had to use its left hand to retrieve bread from the left side and the right hand to retrieve bread from the right side. Each monkey was presented with 18 trials over 2-3 days. There were 6 trials in which both sets of steps were baited, 6 trials in which only the steps on the left were baited, and 6 trials in which only the steps on the right were baited. The trials were presented in a fixed balanced order and the monkey was allowed 5 min to complete each trial. The total number of pieces removed and the time taken to remove all bread pieces ('time-to-clear') were recorded. Monkeys were trained prelesion until they were familiar with the apparatus and could readily retrieve the bread pieces from all the steps. Monkeys were assessed before lesion and at 2, 6, and 10 weeks after the lesion.

Object retrieval task (ORT): This was an adaptation of the ORT first used by Diamond and colleagues $(13,14)$ to measure the motor planning involved in making detour reaches around a transparent barrier to obtain a reward. A correct response required the monkey to be able to control its arm movements, to choose which arm to use on different trials, and to reach around rather than towards a transparent barrier. The latter requires the inhibition of a direct reach towards a visible reward. Training and testing was started approximately 3.5 months 
postlesion. The monkey was placed in a holding chamber which was opaque except for the transparent front face which had a horizontal slot through which the monkey could reach. This face could be covered by an opaque screen between trials. On each trial the monkey was required to reach through the slot to retrieve a piece of marshmallow from a transparent test box $(5 \mathrm{~cm} \times 5 \mathrm{~cm} \times 5 \mathrm{~cm})$. The test box had only one open side which faced towards the monkey's left or right side, in a pseudorandom order across trials. Each monkey was given 22 trials each day and was allowed up to two minutes to retrieve the marshmallow on each trial. Testing continued until the monkey had retrieved at least 20 marshmallow pieces in the 22 trials on each of three consecutive days or for 14 days. Data were analysed for the last three days of testing, i.e. 66 trials. Trials were scored 'perfect' if the reward was taken in one smooth movement using the appropriate arm (left on the left side, right on the right side). All other trials were scored 'imperfect'. For imperfect trials, the number of 'motor errors' and 'barrier reaches' were counted. A motor error occurred when the appropriate arm was directed towards the opening but the food piece was not retrieved because it was dropped or knocked aside. A barrier reach was a reach that encountered the front or the closed side of the box and was, therefore, unsuccessful in retrieving the reward. Repeated barrier reaches were frequently made within one trial and were scored as separate reaches. When the opening was facing the monkey's contralesional side, two types of barrier reach could be made: Type 1, when the appropriate contralesional hand encountered the closed front of the box and Type 2, when the inappropriate ipsilesional hand encountered the closed front or the closed ipsilesional side of the box. Similarly, when the opening was on the ipsilesional side two further types of barrier reach could be made: Type 3, when the inappropriate contralesional hand encountered the closed front or the closed contralesional side of the box and Type 4, when the appropriate ipsilesional hand encountered the closed front of the box (see Fig. 7). 
Testing after $\alpha$-methyl-p-tyrosine (AMPT) administration: At approximately 5 months postlesion, when all the previous behavioural testing had been completed, the monkeys were assessed after administration of AMPT, a tyrosine hydroxylase inhibitor which reduces dopamine levels. The monkeys were given doses of 0 (saline injection), 20, 80, 160, or 240mg/kg AMPT (Sigma, St Louis, MO, USA; dissolved in saline, intramuscularly), 3-4h before being tested on the disability rating scale, head position bias, and the staircase task. AMPT injections were separated by at least 2 days.

\section{Results}

Statistical analysis: Postlesion data were analysed for within- and between-group effects using a repeated measures ANOVA. Where there were significant interactions, post hoc comparisons were made using Bonferroni $t$-tests. Prelesion comparisons between groups were made using $t$-tests.

\section{Histology:}

Nigral cell counts: 6-OHDA lesioned monkeys had a significant loss of TH-IR cells in the substantia nigra ipsilateral to the lesion, with an average cell count of only $46 \%$ of the cell count on the intact side (see Table 2 and Fig 1). Repeated measures ANOVA showed a significant group effect $(\mathrm{F}[1,10]=9.06, \mathrm{P}<0.05)$, a significant effect of side $(\mathrm{F}[1,10]=66.73$, $\mathrm{P}<0.001)$, and a significant group $\mathrm{x}$ side interaction $(\mathrm{F}[1,10]=131.35, \mathrm{P}<0.001)$. Post hoc analysis revealed a significant difference in cell count between the injected and uninjected side 
in the 6-OHDA lesioned monkeys (Bonferroni $t=13.880, \mathrm{P}<0.001$ ) but no difference in the sham lesioned monkeys.

TH-density measures of the striatum: Injection of 6-OHDA resulted in a loss of TH-IR Fibres in the striatum (see Table 3 and Fig 2). The density of TH staining in the injected hemisphere as a percentage of the density in the uninjected hemisphere was $66 \%$ for the caudate nucleus, $72 \%$ for the putamen and $67 \%$ for the nucleus accumbens. Repeated measures ANOVA of the average density measures revealed a significant effect of side $(\mathrm{F}[1,10]=58.64, \mathrm{P}<0.001)$ and a significant group $\mathrm{x}$ side interaction $(\mathrm{F}[1,10]=67.23, \mathrm{P}<0.001)$. Post hoc analysis showed that there was a significant loss of TH staining in the ipsilesional hemisphere in the 6-OHDA injected monkeys compared with staining in the contralesional hemisphere (Bonferroni $t=$ 11.21, $\mathrm{P}<0.001)$.

Behavioural Assessment

Postoperative recovery: All animals were returned to their home cages and cage mates on the day following surgery. They all displayed some change in posture and demeanour (see Disability rating scale and Head position bias) but they were all capable of feeding and selfcare. Despite the changes in skilled hand use on the Staircase task and the Object retrieval task (see below), animals displayed no gross changes in the integration of contralesional arm movements in the automatic behaviours of normal locomotion and other daily activities.

Disability rating scale: Before surgery, the disability rating scores of the 2 groups did not differ significantly $(t=1.853$, n.s.). Following surgery, the 6-OHDA lesioned group had higher disability rating scores than the saline group $(\mathrm{F}[1,10]=12.274, \mathrm{P}<0.01)$ but there was no 
group $\mathrm{x}$ week interaction $(\mathrm{F}[1,10]=3.364$, n.s. $)$, see Fig 3A. The disability consisted mainly of hunched posture, reduced movement and failure to climb. No tremor was observed.

Following AMPT administration, there was a significant difference between groups $(\mathrm{F}[1,10]=$ 19.917, $\mathrm{P}<0.01)$, a significant dose effect $(\mathrm{F}[3,30]=10.254, \mathrm{P}<0.001)$, and a significant group $\mathrm{x}$ dose interaction $(\mathrm{F}[3,30]=6.542, \mathrm{P}<0.05)$. Post hoc analysis revealed significant differences between the 6-OHDA lesioned group and the sham lesioned group at the two highest doses $(160 \mathrm{mg} / \mathrm{kg}$, Bonferroni $t=3.627, \mathrm{P}<0.05$; and $240 \mathrm{mg} / \mathrm{kg}$, Bonferroni $t=4.880, \mathrm{P}<0.01)$, see Fig 3B. AMPT was given approximately 5 months after surgery by which time the 6-OHDA lesioned monkeys did not show any spontaneous impairment (see effect of saline injection in Fig 3B). However, AMPT produced a dose-dependent increase in disability rating scores in the 6-OHDA lesioned monkeys but was without effect on the sham lesioned monkeys.

Head position bias: Before surgery, both groups had similar unbiased scores on the head position bias test ( $t=1.060$, n.s.). Following surgery, the 6-OHDA lesioned monkeys spent more time with their head towards the ipsilesional side compared to sham lesioned monkeys who showed no head position bias $(\mathrm{F}[1,10]=20.191, \mathrm{P}<0.01)$. There was a significant effect of time $(\mathrm{F}[3,30]=16.484, \mathrm{P}<0.001)$ and a time $\mathrm{x}$ group interaction $(\mathrm{F}[3,30]=9.705$, $\mathrm{P}<0.001)$. Post hoc analysis revealed that 6-OHDA lesioned monkeys spent significantly more time with the head turned ipsilesionally than saline animals at weeks one (Bonferroni $t=3.735$, $\mathrm{P}<0.05$ ) and two (Bonferroni $t=3.537, \mathrm{P}<0.05$ ), but not thereafter, see Fig 4A.

Following AMPT administration there was a significant group difference in head position bias $(\mathrm{F}[1,10]=31.35, \mathrm{P}<0.001)$ and a significant group $\mathrm{x}$ dose interaction $(\mathrm{F}[3,10]=4.243$, $\mathrm{P}<0.05)$. Post hoc analysis revealed that 6-OHDA lesioned monkeys turned their heads 
ipsilesionally at all doses of AMPT in comparison to sham lesioned monkeys (80mg/kg, Bonferroni $t=3.692, \mathrm{P}<0.05 ; 160 \mathrm{mg} / \mathrm{kg}$, Bonferroni $t=3.270, \mathrm{P}<0.05 ; 240 \mathrm{mg} / \mathrm{kg}$, Bonferroni $t=4.198, \mathrm{P}<0.01)$, see Fig 4B.

Rotations: Before surgery, neither group had a rotational bias, either spontaneously or after amphetamine or apomorphine administration. Following surgery, none of the monkeys rotated spontaneously. After amphetamine administration, only one 6-OHDA lesioned monkey showed substantial ipsilesional rotation at 2, 6, and 10 weeks. The lesion in this monkey was in the mid-range as assessed by TH-IR histology. The other monkeys in the 6-OHDA group, along with the sham lesioned monkeys, did not rotate substantially after amphetamine administration (data not shown). Following apomorphine administration, in contrast, 6-OHDA lesioned monkeys showed substantial contralesional rotation compared with the sham lesioned monkeys $(\mathrm{F}[1,10]=5.691, \mathrm{P}<0.05)$. Hoever, there was a significant week $\mathrm{x}$ group interaction $(\mathrm{F}[2,20]=7.505, \mathrm{P}<0.01)$ and the apomorphine-induced rotation was lost by week 10 , see Fig 5.

Staircase task: Before surgery, both groups had similar time-to-clear scores and removed the same number of food pieces from the staircase steps. Following surgery, the 6-OHDA lesioned monkeys performed more slowly than the sham lesioned monkeys, but they did not show a specific difficulty with the contralesional arm on this task. ANOVA of the time-to-clear scores revealed a significant lesion $\mathrm{x}$ week interaction $(\mathrm{F}[2,20]=3.624, \mathrm{P}<0.05)$ indicating that in comparison to the sham lesioned monkeys, the 6-OHDA lesioned monkeys had higher time-toclear scores as the weeks progressed, see Fig 6A. 
Following AMPT administration, the 6-OHDA lesioned monkeys had higher time-to-clear scores than the control monkeys $(F[1,10]=5.463, \mathrm{P}<0.05)$, see Fig $6 \mathrm{~B}$. There was no significant group $\mathrm{x}$ dose effect $(\mathrm{F}[3,30]=2.035$, n.s. $)$. However, there was no difference in performance between the contralesional hand and ipsilesional hand in the 6-OHDA lesioned monkeys. 6-OHDA lesioned monkeys retrieved fewer food pieces than did sham controls $(\mathrm{F}[1,10]=6.139, \mathrm{P}<0.05)$. There was an interaction between the dose of AMPT and group in the number of food pieces retrieved $(\mathrm{F}[3,30]=8.401, \mathrm{P}<0.001)$ in that the 6-OHDA lesioned monkeys retrieved fewer food pieces at higher doses of AMPT.

Object retrieval task: There was no difference between the 6-OHDA lesioned monkeys and the sham lesioned monkeys in the number of trials in criterion that were perfect, i.e. in which reward was taken in one smooth movement using the appropriate arm (left arm on the left side, and right arm on the right side). However, amongst the imperfect trials the groups differed in the quality of the responses they made. 6-OHDA lesioned monkeys made significantly more barrier reaches than the sham lesioned monkeys when the box was open on the contralesional side $(\mathrm{F}[1,10]=5.998, \mathrm{P}<0.05)$ comprising both Type 1 errors (barrier reaches with the appropriate contralesional hand, and Type 2 errors (barrier reaches with the inappropriate, ipsilesional hand, see Fig 7. Observation of the 6-OHDA lesioned monkeys indicated that Type 2 errors occurred when they failed to initiate responses with the contralesional hand and, instead, made repeated attempts with the ipsilesional hand to gain access to the reward through the closed side of the box. In contrast, when the opening was on the ipsilesional side, the 6-OHDA lesioned monkeys did not make excess barrier reaches with either hand (see Fig 7). There was no significant difference between the 6-OHDA lesioned monkeys and the sham lesioned monkeys in the number of motor errors made with either arm. 


\section{Discussion}

The purpose of this study was to characterize the behavioural deficits occurring after unilateral injections of 6-OHDA into the caudate, accumbens and putamen in marmoset monkeys. Such injections produced a behavioural syndrome that was of limited duration for some measures, but that was reinstated by the administration of AMPT. 6-OHDA injections reduced the number of TH-IR cells within the substantia nigra to $\sim 46 \%$ and the TH-IR density in the striatum to $\sim 68 \%$ compared to the non-injected side.

Intrastriatal injections of 6-OHDA resulted in a variety of behavioural impairments. The lesioned monkeys displayed parkinsonian signs after surgery as measured by the disability rating scale. By 10 weeks postlesion, the rating score for 6-OHDA lesioned monkeys had recovered to prelesion levels. Treatment with AMPT reinstated a dose dependent deficit on this measure suggesting that functional compensation involving dopamine systems, such as its synthesis or storage, were involved in the recovery seen postlesion.

6-OHDA lesioned monkeys spent substantially more time with their head oriented towards their ipsilesional side, with the highest level being seen at one week postlesion. This impairment returned to baseline levels by 6 weeks postlesion. Head position bias has been shown to be a reliable measure of sensorimotor deficits in DA depleted marmosets. Annett and colleagues (1) reported that the greatest levels of head bias were seen one week after 6OHDA lesions of the substantia nigra after which there was a gradual decline in the levels of bias, although the head bias did not return to prelesion levels in the case of these near complete dopamine lesions. In the present study the administration of AMPT reinstated head 
bias to levels seen at one week postlesion. This indicates that along with the disability rating scale, head position bias is a useful measure of behavioural deficit but sensitive to recovery.

The quantification of rotational behaviour occurring after unilateral lesions of the DA system is a standard tool for assessing dopamine loss within the nigrostriatal system (52). In the present study, 6-OHDA lesioned monkeys rotated contralesionally after apomorphine administration at 2 and 6 weeks postlesion but had returned to baseline levels by week 10 postlesion. They did not, however, rotate spontaneously or in response to amphetamine. This differs from the effects of 6-OHDA lesions of the substantia nigra, nigrostriatal bundle or basal ganglia in rats or the effects of nigrostriatal bundle lesions in marmosets, where both amphetamine and apomorphine induced rotations are seen. A possible source for this discrepancy may lie in the level of locomotor activity resulting from injections of these two drugs. Unlesioned marmosets show a marked increase in locomotion in response to injections of apomorphine but not in response to injections of amphetamine over a wide dose range (45). If the level of locomotor activity contributes to the rate of rotation, particularly at lower lesion sizes, this may produce differential results. A rotational response to amphetamine may only be seen in marmosets with the very large unilateral DA depletions achieved by bundle nigrostriatal lesions.

The staircase task has previously been used in marmoset monkeys to assess skilled sensorimotor ability $(24,27) .6-$ OHDA lesioned monkeys in the present study were slower and somewhat less successful at retrieving rewards in this task especially under AMPT, although there was no significant effect of side and, therefore, of hand. Unilateral 6-OHDA partial lesion studies in rats have shown a variable contralesional paw deficit, perhaps dependent on lesion size $(2,34,34,47)$. Recovery of paw deficits may also occur (46). Ipsilesional hand 
deficits may occur in sensorimotor tasks, particularly with the complete unilateral lesion model (e.g. $(37,38,41)$. Miklyaeva and colleagues (37) reported that ipsilesional deficits in a reaching task may occur as a consequence of postural abnormalities associated with unilateral nigrostriatal DA loss.

The object retrieval task was originally designed to measure deficits in human infants and rhesus monkeys (13). The task assesses the degree to which monkeys are able to make detour reaches around a transparent barrier, an ability shown to be sensitive to DA loss (55). In order to succeed monkeys need to refrain from making inappropriate arm movements that are directed at the barrier. Taylor and colleagues $(55,56)$ have shown that monkeys treated with MPTP, that are asymptomatic for gross motor deficits, make more inappropriate responses directed at the barrier compared to untreated controls. In the present experiment, 6-OHDA lesioned monkeys made more barrier errors when the open side of the test box was facing their contralesional side. Specifically, when the box was open on the contralesional side, 6-OHDA lesioned monkeys had poor motor control of their contralesional hand and made incorrect reaches towards the front barrier with that hand. However, they made even more barrier reaches in this position with their ipsilesional hand. This suggests that these monkeys were reluctant to use the appropriate contralesional hand, but were motivated enough to resort to using their inappropriate ipsilesional hand, thereby making a substantial number of barrier reaches with that hand. The increase in use of the ipsilesional hand is probably a natural consequence of this reluctance to use the contralesional hand rather than an effect of the lesion on the motor control of the ipsilesional arm. It is noteworthy that at three and a half months postlesion, at a time when behavioural recovery had taken place on other measures, the ORT was still able to detect sensorimotor behavioural deficits. 
The tyrosine hydroxylase inhibitor AMPT reinstated or exacerbated deficits on the disability rating scale, head position bias and the staircase task. This suggests that compensatory mechanisms involving dopamine were involved in the recovery seen postlesion. Axonal sprouting of the remaining dopamine cells, an increase in TH activity and an increase in DA turnover have been observed to occur in rats after 6-OHDA lesions $(18,61)$, and may be the basis of the recovery seen in the present study.

The present study has shown that intrastriatal 6-OHDA lesions of the DA system in marmoset monkeys result in a variety of behavioural deficits which parallel many of the deficits seen in rodent studies. A number of these deficits in marmosets are prone to recovery but are reinstated by the TH inhibitor AMPT. The development of a functionally more stable intrastriatal 6-OHDA model may require a greater level of cell loss than that seen in the present experiment. Nonetheless the intrastriatal 6-OHDA lesion model in monkeys has potential for assessing the effects of treatments for PD that aim to curtail disease progression, such as continuous GDNF delivery via viral vectors. 


\section{Acknowledgements}

We are grateful to Professor Anders Björklund and Dr Deniz Kirik for advice, and other members of their laboratory at Lund University, Sweden, for technical assistance and advice in respect of histological analysis. AE and LEA were supported by the Wellcome Trust. HFB and RMR were supported by the Medical Research Council. 


\section{Legends}

Fig 1. Coronal sections at $3 \mathrm{AP}$ levels through the substantia nigra of a marmoset following unilateral injection of 6-OHDA in the basal ganglia. The injected side is on the left of the figure. These digital images were prepared by scanning the microscope slides directly. There is a reduction in TH-IR cells on the left hand side. See Table 2 for quantification of lesion. Bar $=$ $1 \mathrm{~mm}$.

Fig 2. Coronal sections of 3 AP levels through whole brain of a marmoset following unilateral injection of 6-OHDA in the basal ganglia. The injected side is on the left of the figure. These digital images were prepared by scanning the microscope slides directly. There is a reduction in TH-IR staining in the caudate, nucleus accumbens and putamen on the left hand side. See Table 3 for quantification. Bar $=5 \mathrm{~mm}$.

Fig 3. Effects of lesion on disability rating. A: Disability rating before and after lesion. B: Disability rating 5 months after lesion but 3-4 hours after administration of AMPT. Open columns: saline injected group. Filled columns: 6-OHDA injected group. ${ }^{*} \mathrm{P}<0.05$, ${ }^{*} * \mathrm{P}<0.01$.

Fig 4. Effects of lesion on head position bias. A: Head bias before and after lesion expressed as mean number of ipsilesional minus contralesional head turns. B: Head bias 5 months after lesion but 3-4 hours after administration of AMPT. Open columns: saline injected group. Filled columns: 6-OHDA injected group. ${ }^{*} \mathrm{P}<0.05, * * \mathrm{P}<0.01$. 
Fig 5. Effect of apomorphine administration on rotation before and after lesion expressed as mean number of ipsilesional minus contralesional complete rotations. Open columns: saline injected group. Filled columns: 6-OHDA injected group.

Fig 6. Effect of lesion on time to clear all rewards on the staircase task. A: Before and after lesion. B: 5 months after lesion but 3-4 hours after administration of AMPT. Open columns: saline injected group. Filled columns: 6-OHDA injected group.

Fig 7. Effect of lesion on the mean number of barrier reaches on the object retrieval task. The blob in the cartoon indicates lesion side. When the opening of the box was on the contralesional side, errors could be made with the contralesional arm (Type 1 errors) or the ipsilesional arm (Type 2 errors). When the opening of the box was on the ipsilesional side, errors could be made with the contralesional arm (Type 3 errors) or the ipsilesional arm (Type 4 errors). Open columns: saline injected group. Filled columns: 6-OHDA injected group. 
Table 1 Lesion co-ordinates ( $\mathrm{mm}$ from ear bar zero)

\begin{tabular}{cccc}
\hline Site & Anterior/Posterior & Medial/Lateral & Dorsal/Ventral \\
\hline 1 & 9.0 & $+/-6.0$ & 10.5 \\
2 & 8.0 & $+/-6.2$ & 10.5 \\
3 & 7.0 & $+/-6.5$ & 10.0 \\
4 & 12.5 & $+/-3.0$ & 12.0 \\
5 & 11.5 & $+/-4.4$ & 10.0 \\
6 & 10.5 & $+/-2.6$ & 12.2 \\
7 & 13.0 & $+/-2.0$ & 9.2 \\
8 & 12.0 & $+/-2.0$ & 9.2 \\
9 & 11.0 & $+/-2.2$ & 9.2 \\
\hline
\end{tabular}

Coordinates based on stereotaxic atlas of Stephan et al. (53) 
Table 2 Cell counts in substantia nigra ( +/- sem)

Uninjected side $\quad$ Injected side $\quad \%$ of uninjected side

Saline injected

$33016+/-2547$

$36461+/-1760$

110

6-OHDA injected

$38155+/-1293$

$17607+/-1147$

46

The coefficient of errors for estimates of total cell counts were $<0.1$ for each group/side as calculated by the method of Gundersen and Jenson (22). 
Table 3 Density of tyrosine hydroxylase staining (density units +/- sem)

Uninjected side $\quad$ Injected side $\%$ of uninjected side

Saline injected

$\begin{array}{lccc}\text { Caudate } & 67.19+/-1.63 & 68.87+/-1.73 & 102.5 \\ \text { Putamen } & 67.79+/-2.30 & 69.36+/-2.19 & 102.3 \\ \text { N. Accumbens } & 65.17+/-3.03 & 64.24+/-2.88 & 98.6 \\ & & & \text { Mean }=101.1\end{array}$

6-OHDA injected

$\begin{array}{lccc}\text { Caudate } & 70.20+/-5.48 & 46.23+/-7.08 & 65.9 \\ \text { Putamen } & 72.73+/-5.09 & 52.23+/-5.09 & 71.8 \\ \text { N. Accumbens } & 70.06+/-4.74 & 46.98+/-5.11 & 67.1 \\ & & \text { Mean }=68.3\end{array}$




\section{References}

1. Annett, L. E., D. C. Rogers, T. D. Hernandez, and S. B. Dunnett. 1992. Behavioural analysis of unilateral monoamine depletion in the marmoset. Brain. 115: 825-856.

2. Barneoud, P., E. Descombris, N. Aubin, and D. N. Abrous. 2000. Evaluation of simple and complex sensorimotor behaviours in rats with a partial lesion of the dopaminergic nigrostriatal system. Eur.J.Neurosci. 12: 322-336.

3. Beck, K. D., J. Valverde, T. Alexi, K. Poulsen, B. Moffat, R. A. Vandlen, A. Rosenthal, and F. Hefti. 1995. Mesencephalic dopaminergic neurons protected by GDNF from axotomy-induced degeneration in the adult brain. Nature. 373: 339-341.

4. Bjorklund, A. and O. Lindvall. 2000. Parkinson disease gene therapy moves toward the clinic. Nat.Med. 6: 1207-1208.

5. Bjorklund, A., C. Rosenblad, C. Winkler, and D. Kirik. 1997. Studies on neuroprotective and regenerative effects of GDNF in a partial lesion model of Parkinson's disease. Neurobiol.Dis. 4: 186-200.

6. Blanchet, S., R. M. Marie, F. Dauvillier, B. Landeau, K. Benali, F. Eustache, and C. Chavoix. 2000. Cognitive processes involved in delayed non-matching-to-sample performance in Parkinson's disease. Eur.J.Neurol. 7: 473-483.

7. Brundin, P., O. Pogarell, P. Hagell, P. Piccini, H. Widner, A. Schrag, A. Kupsch, L. Crabb, P. Odin, B. Gustavii, A. Bjorklund, D. J. Brooks, C. D. Marsden, W. H. Oertel, N. P. Quinn, S. Rehncrona, and O. Lindvall. 2000. Bilateral caudate and putamen grafts 
of embryonic mesencephalic tissue treated with lazaroids in Parkinson's disease. Brain. 123: $1380-1390$.

8. Carli, M., G. H. Jones, and T. W. Robbins. 1989. Effects of unilateral dorsal and ventral striatal dopamine depletion on visual neglect in the rat: a neural and behavioural analysis. Neurosci. 29: 309-327.

9. Chio, A., C. Magnani, and D. Schiffer. 1998. Prevalence of Parkinson's disease in Northwestern Italy: comparison of tracer methodology and clinical ascertainment of cases. Mov.Disord. 13: 400-405.

10. Connor, B., D. A. Kozlowski, J. R. Unnerstall, J. D. Elsworth, J. L. Tillerson, T. Schallert, and M. C. Bohn. 2001. Glial cell line-derived neurotrophic factor (GDNF) gene delivery protects dopaminergic terminals from degeneration. Exp.Neurol. 169: 8395.

11. Costa, S., M. M. Iravani, R. K. Pearce, and P. Jenner. 2001. Glial cell line-derived neurotrophic factor concentration dependently improves disability and motor activity in MPTP-treated common marmosets. Eur.J.Pharmacol. 412: 45-50.

12. Damier, P., E. C. Hirsch, Y. Agid, and A. M. Graybiel. 1999. The substantia nigra of the human brain. II. Patterns of loss of dopamine-containing neurons in Parkinson's disease. Brain. 122: 1437-1448.

13. Diamond, A. and Goldman-Rakic P. 1985. Evidence for involvement of prefrontal cortex in cognitive changes during the first year of life: comparison of performance of human infants and rhesus monkeys on a detour task with transparent barrier. Soc.Neurosci.Abs. 11: 832-832. 
14. Diamond, A., S. Zola-Morgan, and L. R. Squire. 1989. Successful performance by monkeys with lesions of the hippocampal formation on $\mathrm{AB}$ and object retrieval, two tasks that mark developmental changes in human infants. Behav.Neurosci. 103: 526-537.

15. Durif, F., J. J. Lemaire, B. Debilly, and G. Dordain. 1999. Acute and chronic effects of anteromedial globus pallidus stimulation in Parkinson's disease. J.Neurol.Neurosurg.Psychiatry. 67: 315-322.

16. Errea, J. M., J. R. Ara, C. Aibar, and J. de-Pedro-Cuesta. 1999. Prevalence of Parkinson's disease in lower Aragon, Spain. Mov.Disord. 14: 596-604.

17. Fahn, S. 1997. An overview of novel therapies for Parkinson's disease. Exp.Neurol. 144: 21-23.

18. Finkelstein, D. I., D. Stanic, C. L. Parish, D. Tomas, K. Dickson, and M. K. Horne. 2000. Axonal sprouting following lesions of the rat substantia nigra. Neurosci. 97: 99112.

19. Gash, D. M., Z. Zhang, A. Ovadia, W. A. Cass, A. Yi, L. Simmerman, D. Russell, D. Martin, P. A. Lapchak, F. Collins, B. J. Hoffer, and G. A. Gerhardt. 1996. Functional recovery in parkinsonian monkeys treated with GDNF. Nature. 380: 252-255.

20. Gelb, D. J., E. Oliver, and S. Gilman. 1999. Diagnostic criteria for Parkinson disease. Arch.Neurol. 56: 33-39.

21. Gerhardt, G. A., W. A. Cass, P. Huettl, S. Brock, Z. Zhang, and D. M. Gash. 1999. GDNF improves dopamine function in the substantia nigra but not the putamen of unilateral MPTP-lesioned rhesus monkeys. Brain Res. 817: 163-171. 
22. Gundersen, H. J. C. and E. B. Jensen. 1987. The efficiency of systematic sampling in stereology and its prediction. J.Microsc. 147: 229-263.

23. Hagell, P., A. Schrag, P. Piccini, M. Jahanshahi, R. Brown, S. Rehncrona, H. Widner, P. Brundin, J. C. Rothwell, P. Odin, G. K. Wenning, P. Morrish, B. Gustavii, A. Bjorklund, D. J. Brooks, C. D. Marsden, N. P. Quinn, and O. Lindvall. 1999. Sequential bilateral transplantation in Parkinson's disease: effects of the second graft. Brain. 122: 11211132.

24. Henderson, J. M., L. E. Annett, E. M. Torres, and S. B. Dunnett. 1998. Behavioural effects of subthalamic nucleus lesions in the hemiparkinsonian marmoset (Callithrix jacchus). Eur.J.Neurosci. 10: 689-698.

25. Hirsch, E. C., A. M. Graybiel, and Y. Agid. 1989. Selective vulnerability of pigmented dopaminergic neurons in Parkinson's disease. Acta Neurol.Scand.Suppl. 126: 19-22.

26. Kawamoto, Y., S. Nakamura, A. Matsuo, I. Akiguchi, and H. Shibasaki. 2000. Immunohistochemical localization of glial cell line-derived neurotrophic factor in the human central nervous system. Neurosci. 100: 701-712.

27. Kendall, A. L., F. David, G. Rayment, E. M. Torres, L. E. Annett, and S. B. Dunnett. 2000. The influence of excitotoxic basal ganglia lesions on motor performance in the common marmoset. Brain. 123: 1442-1458.

28. Kirik, D., B. Georgievska, C. Rosenblad, and A. Bjorklund. 2001. Delayed infusion of GDNF promotes recovery of motor function in the partial lesion model of Parkinson's disease. Eur.J.Neurosci. 13: 1589-1599. 
29. Kirik, D., C. Rosenblad, and A. Bjorklund. 1998. Characterization of behavioral and neurodegenerative changes following partial lesions of the nigrostriatal dopamine system induced by intrastriatal 6-hydroxydopamine in the rat. Exp.Neurol. 152: 259-277.

30. Kirik, D., C. Rosenblad, and A. Bjorklund. 2000. Preservation of a functional nigrostriatal dopamine pathway by GDNF in the intrastriatal 6-OHDA lesion model depends on the site of administration of the trophic factor. Eur.J.Neurosci. 12: 38713882.

31. Kirik, D., C. Rosenblad, A. Bjorklund, and R. J. Mandel. 2000. Long-term rAAVmediated gene transfer of GDNF in the rat Parkinson's model: intrastriatal but not intranigral transduction promotes functional regeneration in the lesioned nigrostriatal system. J.Neurosci. 20: 4686-4700.

32. Kordower, J. H., M. E. Emborg, J. Bloch, S. Y. Ma, Y. Chu, L. Leventhal, J. McBride, E. Y. Chen, S. Palfi, B. Z. Roitberg, W. D. Brown, J. E. Holden, R. Pyzalski, M. D. Taylor, P. Carvey, Z. Ling, D. Trono, P. Hantraye, N. Deglon, and P. Aebischer. 2000. Neurodegeneration prevented by lentiviral vector delivery of GDNF in primate models of Parkinson's disease. Science. 290: 767-773.

33. Lapchak, P. A., D. M. Gash, S. Jiao, P. J. Miller, and D. Hilt. 1997. Glial cell linederived neurotrophic factor: a novel therapeutic approach to treat motor dysfunction in Parkinson's disease. Exp.Neurol. 144: 29-34.

34. Lee, C. S., H. Sauer, and A. Bjorklund. 1996. Dopaminergic neuronal degeneration and motor impairments following axon terminal lesion by instrastriatal 6-hydroxydopamine in the rat. Neurosci. 72: 641-653. 
35. MacDonald, B. K., O. C. Cockerell, J. W. Sander, and S. D. Shorvon. 2000. The incidence and lifetime prevalence of neurological disorders in a prospective communitybased study in the UK. Brain. 123: 665-676.

36. Mandel, R. J., R. O. Snyder, and S. E. Leff. 1999. Recombinant adeno-associated viral vector-mediated glial cell line-derived neurotrophic factor gene transfer protects nigral dopamine neurons after onset of progressive degeneration in a rat model of Parkinson's disease. Exp.Neurol. 160: 205-214.

37. Miklyaeva, E. I., E. Castaneda, and I. Q. Whishaw. 1994. Skilled reaching deficits in unilateral dopamine-depleted rats: impairments in movement and posture and compensatory adjustments. J.Neurosci. 14: 7148-7158.

38. Montoya, C. P., S. Astell, and S. B. Dunnett. 1990. Effects of nigral and striatal grafts on skilled forelimb use in the rat. Prog.Brain Res. 82: 459-466.

39. Montoya, C. P., L. J. Campbell-Hope, K. D. Pemberton, and S. B. Dunnett. 1991. The "staircase test": a measure of independent forelimb reaching and grasping abilities in rats. J.Neurosci.Methods. 36: 219-228.

40. Nosrat, C. A., A. Tomac, E. Lindqvist, S. Lindskog, C. Humpel, I. Stromberg, T. Ebendal, B. J. Hoffer, and L. Olson. 1996. Cellular expression of GDNF mRNA suggests multiple functions inside and outside the nervous system. Cell Tissue Res. 286: 191-207.

41. Olsson, M., G. Nikkhah, C. Bentlage, and A. Bjorklund. 1995. Forelimb akinesia in the rat Parkinson model: differential effects of dopamine agonists and nigral transplants as assessed by a new stepping test. J.Neurosci. 15: 3863-3875. 
42. Owen, A. M., J. L. Iddon, J. R. Hodges, B. A. Summers, and T. W. Robbins. 1997. Spatial and non-spatial working memory at different stages of Parkinson's disease. Neuropsychologia. 35: 519-532.

43. Przedborski, S., M. Levivier, H. Jiang, M. Ferreira, V. Jackson-Lewis, D. Donaldson, and D. M. Togasaki. 1995. Dose-dependent lesions of the dopaminergic nigrostriatal pathway induced by intrastriatal injection of 6-hydroxydopamine. Neurosci. 67: 631647.

44. Rascol, O., D. J. Brooks, A. D. Korczyn, P. P. De-Deyn, C. E. Clarke, and A. E. Lang. 2000. A five-year study of the incidence of dyskinesia in patients with early Parkinson's disease who were treated with ropinirole or levodopa. 056 Study Group. N.Engl.J.Med. 342: 1484-1491.

45. Ridley, R. M., H. F. Baker, and T. J. Crow. 1980. Behavioral effects of amphetamines and related stimulants: the importance of species differences as demonstrated by a study in the marmoset. In Amphetamine and related stimulants: chemical, biological, clinical, and sociological aspects (J. Caldwell, Ed.), pp. 97-116. CRC Press, Inc, Boca Raton, Florida

46. Rodter, A., C. Winkler, M. Samii, and G. Nikkhah. 2000. Complex sensorimotor behavioral changes after terminal striatal 6-OHDA lesion and transplantation of dopaminergic embryonic micrografts. Cell Transplant. 9: 197-214.

47. Roedter, A., C. Winkler, M. Samii, G. F. Walter, A. Brandis, and G. Nikkhah. 2001. Comparison of unilateral and bilateral intrastriatal 6-hydroxydopamine-induced axon 
terminal lesions: evidence for interhemispheric functional coupling of the two nigrostriatal pathways. J.Comp.Neurol. 432: 217-229.

48. Rosenblad, C., D. Kirik, and A. Bjorklund. 2000. Sequential administration of GDNF into the substantia nigra and striatum promotes dopamine neuron survival and axonal sprouting but not striatal reinnervation or functional recovery in the partial 6-OHDA lesion model. Exp.Neurol. 161: 503-516.

49. Rosenblad, C., D. Kirik, B. Devaux, B. Moffat, H. S. Phillips, and A. Bjorklund. 1999. Protection and regeneration of nigral dopaminergic neurons by neurturin or GDNF in a partial lesion model of Parkinson's disease after administration into the striatum or the lateral ventricle. Eur.J.Neurosci. 11: 1554-1566.

50. Rosenblad, C., A. Martinez-Serrano, and A. Bjorklund. 1998. Intrastriatal glial cell linederived neurotrophic factor promotes sprouting of spared nigrostriatal dopaminergic afferents and induces recovery of function in a rat model of Parkinson's disease. Neurosci. 82: 129-137.

51. Sauer, H. and W. H. Oertel. 1994. Progressive degeneration of nigrostriatal dopamine neurons following intrastriatal terminal lesions with 6-hydroxydopamine: a combined retrograde tracing and immunocytochemical study in the rat. Neurosci. 59: 401-415.

52. Schwarting, R. K. and J. P. Huston. 1996. The unilateral 6-hydroxydopamine lesion model in behavioral brain research. Analysis of functional deficits, recovery and treatments. Prog.Neurobiol. 50: 275-331.

53. Stephan, H., G. Baron, and Schwerdtfeger W.K. 1980. The brain of the common marmoset (Callithrix jacchus) A Stereotaxic Atlas. Springer-Verlag, Berlin 
54. Stern, M. B. and A. Freese. 1997. Parkinson's disease: the case for novel treatment strategies. Exp.Neurol. 144: 2-3.

55. Taylor, J. R., J. D. Elsworth, R. H. Roth, J. R. Sladek, and D. E. Redmond. 1990. Cognitive and motor deficits in the acquisition of an object retrieval/detour task in MPTP-treated monkeys. Brain. 113: 617-637.

56. Taylor, J. R. and R. H. Roth. 1990. Cognitive and motor deficits in the performance of an object retrieval task with a barrier-detour in monkeys (Cercopithecus aethiops sabaeus) treated with MPTP: long-term performance and effect of transparency of the barrier. Behav.Neurosci. 104: 564-576.

57. Tseng, J. L., E. E. Baetge, A. D. Zurn, and P. Aebischer. 1997. GDNF reduces druginduced rotational behavior after medial forebrain bundle transection by a mechanism not involving striatal dopamine. J.Neurosci. 17: 325-333.

58. West, M. J., L. Slomianka, and H. J. Gundersen. 1991. Unbiased stereological estimation of the total number of neurons in thesubdivisions of the rat hippocampus using the optical fractionator. Anat.Rec. 231: 482-497.

59. Winkler, C., H. Sauer, C. S. Lee, and A. Bjorklund. 1996. Short-term GDNF treatment provides long-term rescue of lesioned nigral dopaminergic neurons in a rat model of Parkinson's disease. J.Neurosci. 16: 7206-7215.

60. Zhang, Z., Y. Miyoshi, P. A. Lapchak, F. Collins, D. Hilt, C. Lebel, R. Kryscio, and D. M. Gash. 1997. Dose response to intraventricular glial cell line-derived neurotrophic factor administration in parkinsonian monkeys. J.Pharmacol.Exp.Ther. 282: 1396-1401. 
61. Zigmond, M. J., A. L. Acheson, M. K. Stachowiak, and E. M. Stricker. 1984.

Neurochemical compensation after nigrostriatal bundle injury in an animal model of preclinical parkinsonism. Arch.Neurol. 41: 856-861. 
Figures

Fig 1

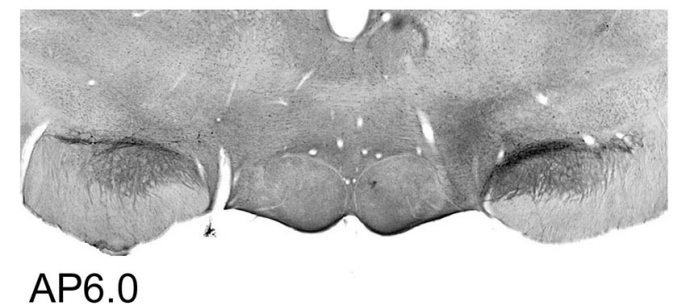

AP6.0

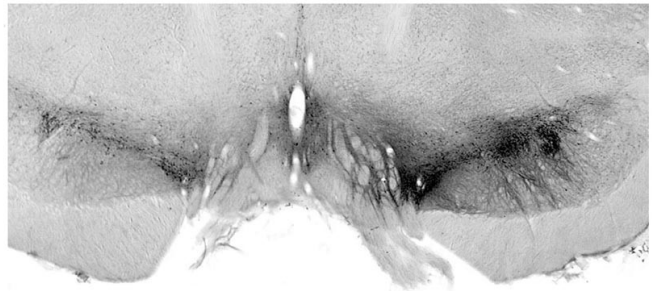

AP5.5

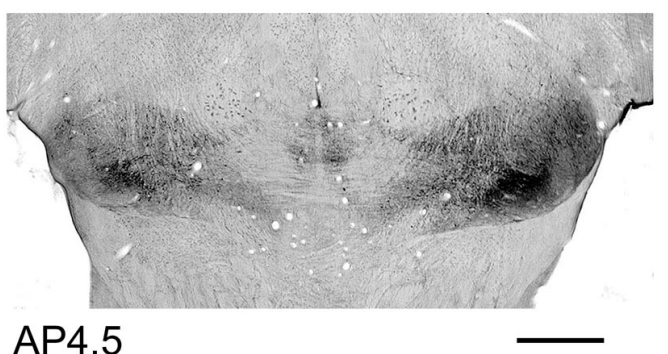

Fig 2
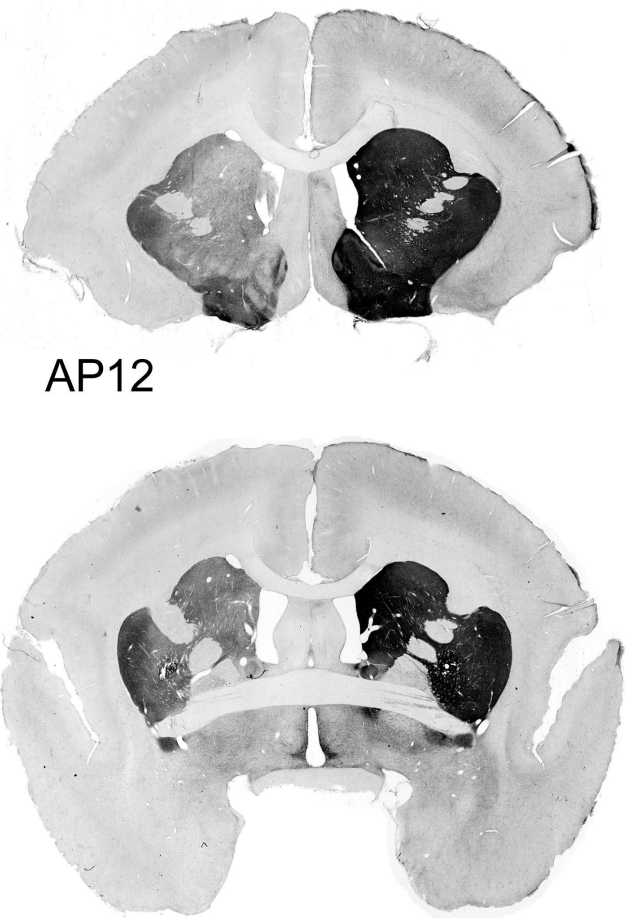

AP9.5

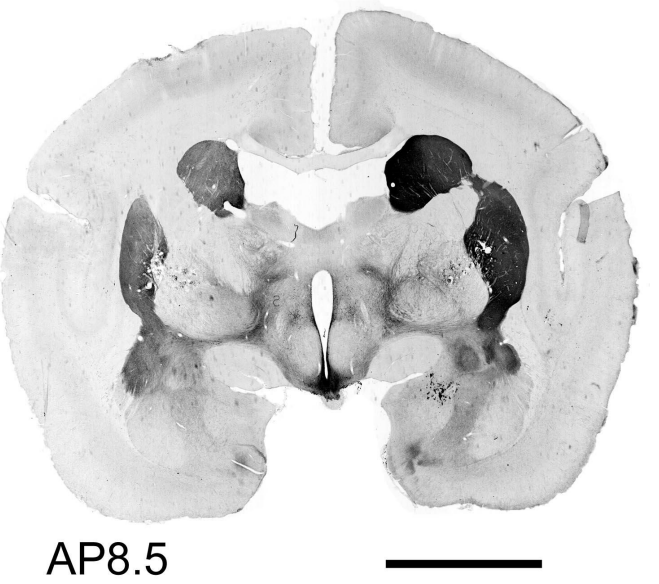


Fig 3

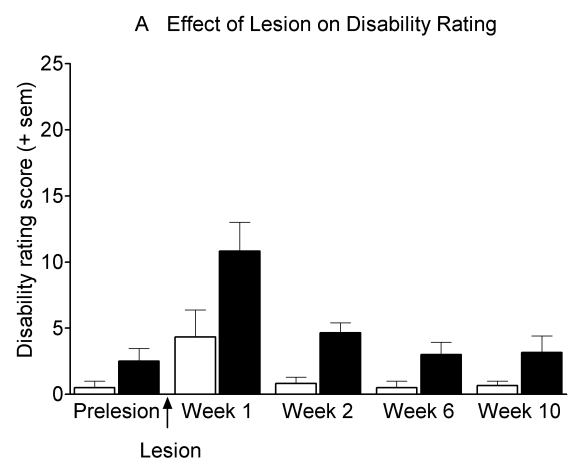

B Effect of AMPT on Disability Rating

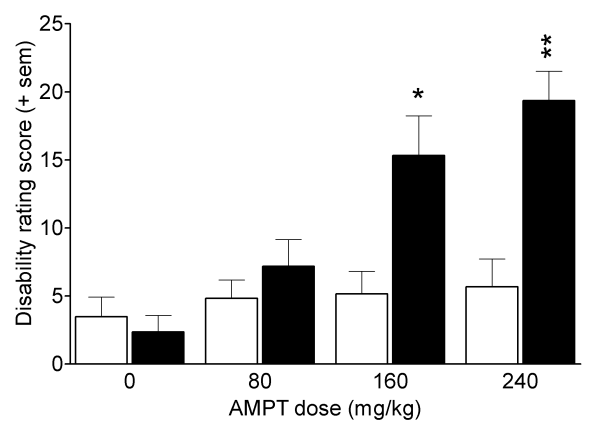

Fig 4

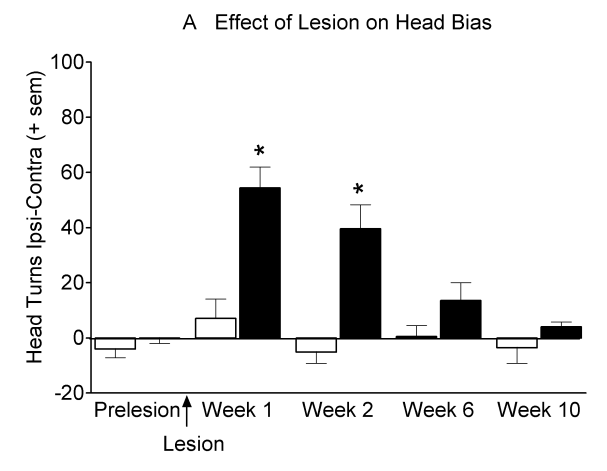

B Effect of AMPT on Head Position Bias

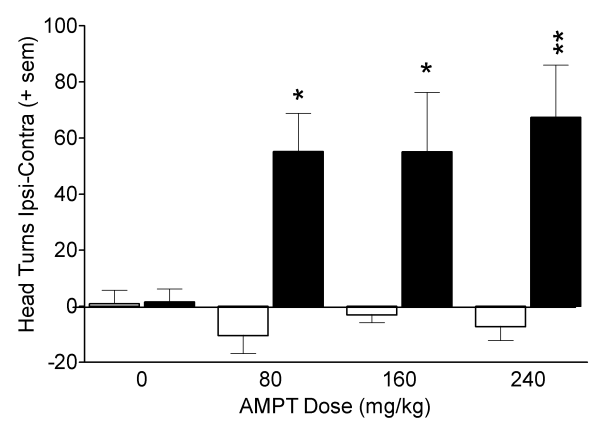

Fig 5

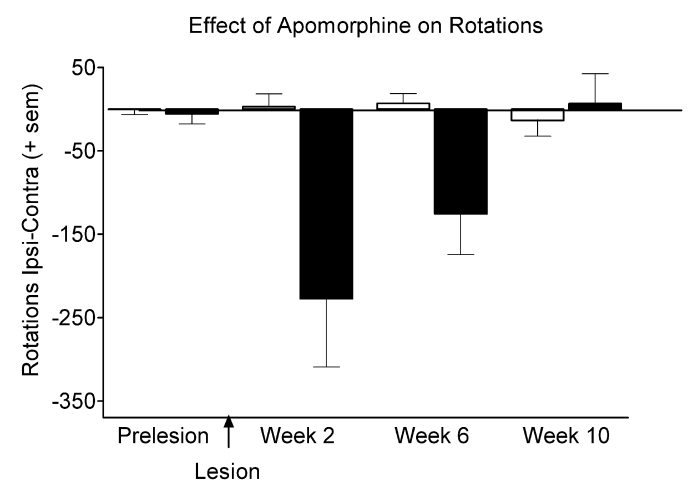


Fig 6
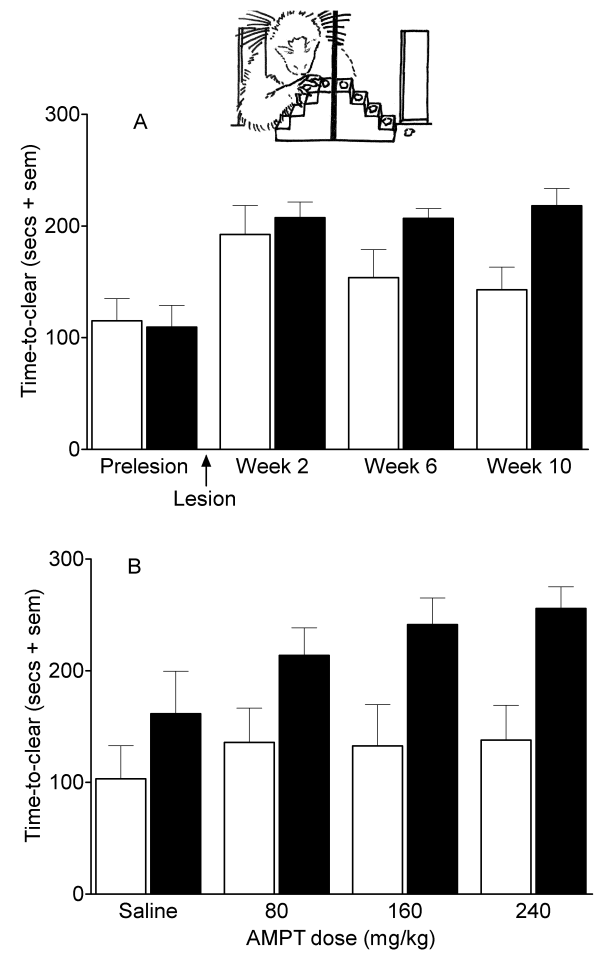

Fig 7
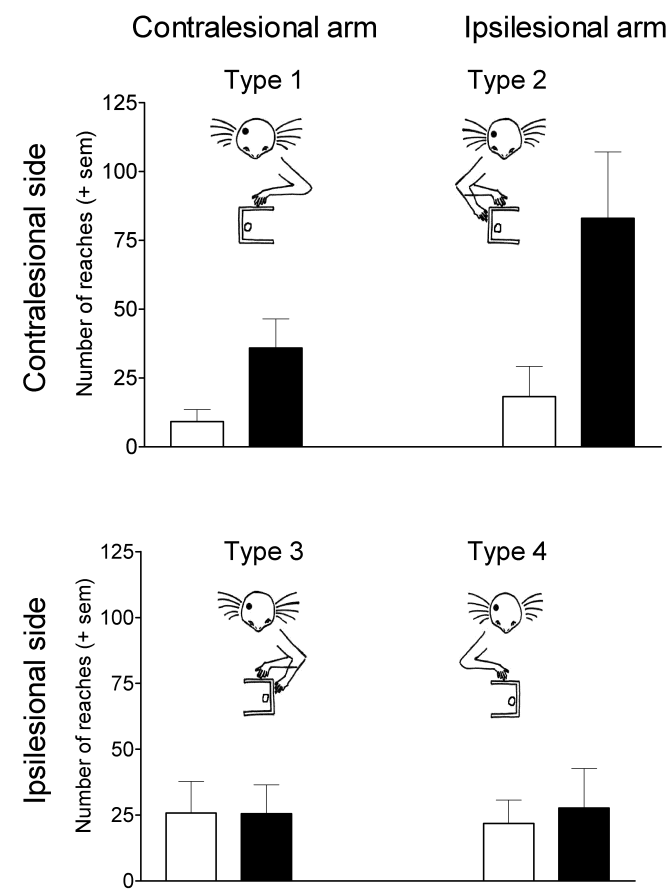